\title{
Measuring the electric dipole moment of the electron in BaF
}

\author{
The NL-eEDM collaboration: Parul Aggarwal ${ }^{1,2}$, Hendrick L. Bethlem ${ }^{3}$, Anastasia Borschevsky ${ }^{1,2}$, \\ Malika Denis ${ }^{1,2}$, Kevin Esajas ${ }^{1,2}$, Pi A.B. Haase ${ }^{1,2}$, Yongliang Hao ${ }^{1,2}$, Steven Hoekstra ${ }^{1,2, a}$, \\ Klaus Jungmann ${ }^{1,2}$, Thomas B. Meijknecht ${ }^{1,2}$, Maarten C. Mooij ${ }^{2,3}$, Rob G.E. Timmermans ${ }^{1,2}$, \\ Wim Ubachs ${ }^{3}$, Lorenz Willmann ${ }^{1,2}$, and Artem Zapara ${ }^{1,2}$ \\ ${ }^{1}$ Van Swinderen Institute for Particle Physics and Gravity (VSI), University of Groningen, Groningen, \\ The Netherlands \\ 2 Nikhef, National Institute for Subatomic Physics, Science Park 105, 1098 XG Amsterdam, The Netherlands \\ 3 LaserLaB, Department of Physics and Astronomy, Vrije Universiteit, De Boelelaan 1081, 1081 HV Amsterdam, \\ The Netherlands
}

Received 25 April 2018 / Received in final form 28 August 2018

Published online 20 November 2018

(C) The Author(s) 2018. This article is published with open access at Springerlink.com

\begin{abstract}
We investigate the merits of a measurement of the permanent electric dipole moment of the electron $(e \mathrm{EDM})$ with barium monofluoride molecules, thereby searching for phenomena of $C P$ violation beyond those incorporated in the standard model (SM) of particle physics. Although the BaF molecule has a smaller enhancement factor in terms of the effective electric field than other molecules used in current studies ( $\mathrm{YbF}, \mathrm{ThO}$ and $\mathrm{ThF}^{+}$), we show that a competitive measurement is possible by combining Starkdeceleration, laser-cooling and an intense primary cold source of BaF molecules. With the long coherent interaction times obtainable in a cold beam of BaF, a sensitivity of $5 \times 10^{-30} \mathrm{e} \cdot \mathrm{cm}$ for an $e$ EDM is feasible. We describe the rationale, the challenges and the experimental methods envisioned to achieve this target.
\end{abstract}

\section{Introduction}

The discovery of the Higgs boson [1,2] at CERN's Large Hadron Collider (LHC) started a new era in particle physics. The LHC will continue to test the standard model (SM) by mapping out the properties of the Higgs boson with increasing precision. Moreover, now that we know that the Higgs boson exists, unanswered questions in particle physics and cosmology come into focus. The Higgs field is responsible for the electroweak-symmetry breaking in the very early Universe. The most natural explanation of why this breaking occurs at a scale of $100 \mathrm{GeV}$ predicts new particles with masses in the $\mathrm{TeV}$ range, some of which could make up the dark matter in the Universe [3]. Leptons and quarks acquire mass via the Higgs field, such that particles and antiparticles have slightly different weak interactions. This symmetry breaking between matter and antimatter is called $C P$ violation, where $C$ denotes charge conjugation and $P$ parity. $C P$ violation within the SM, however, is insufficient by orders of magnitude to explain the matter-antimatter asymmetry in the Universe. This failure of the SM provides a major motivation to search for new sources of $C P$ violation at the $\mathrm{TeV}$ scale. This is the mission of, for example, the $\mathrm{LHCb}$ experiment [4].

Experiments that search for permanent electric dipole moments (EDMs) are complementary to, and compete

\footnotetext{
${ }^{\mathrm{a}}$ e-mail: s.hoekstra@rug.nl
}

with searches of $C P$ violation with antiparticles at highenergy colliders $[5,6]$. A nonzero electron $\operatorname{EDM}(e \mathrm{EDM})$ implies that the electron effectively has an aspherical charge distribution along its spin axis, which is forbidden by time-reversal $(T)$ invariance. According to the $C P T$ theorem of quantum field theory, $T$ violation is equivalent to $C P$ violation. In the $\mathrm{SM}$ the $e \mathrm{EDM}$ is zero, up to the three-loop level. Its predicted value [5] is $d_{e}=\mathcal{O}\left(10^{-38}\right)$ e.cm, which is experimentally far out of reach. However, extensions of the SM invariably predict much larger values, and thus provide a window of opportunity for experimental searches. The reason is that in such theories new heavy particles are introduced that have interactions with $C P$-violating quantum-mechanical phases, giving rise to an $e \mathrm{EDM}$ at the one- or two-loop levels, or even at tree level [7]. For instance, supersymmetry (SUSY), a conjectured symmetry between bosons and fermions, predicts an $e \mathrm{EDM}$ due to interactions with virtual selectrons and photinos, the SUSY partners of the electron and photon at the one-loop level. If such new particles with $\mathrm{TeV}$-scale masses exist, the $e \mathrm{EDM}$ will have a measurable value in the ongoing and upcoming experiments.

The relation between the masses of the yet to be discovered particles and the $e \mathrm{EDM}$ value in different types of models is illustrated in Figure 1. The blue and green lines show the limits on the $e \mathrm{EDM}$ set by experiments with molecular beams of $\mathrm{YbF}[8]$ and $\mathrm{ThO}$ [9], respectively. The purple line shows the limit aimed at in our experiment with $\mathrm{BaF}$. It is obvious from Figure 1 that existing $e \mathrm{EDM}$ 


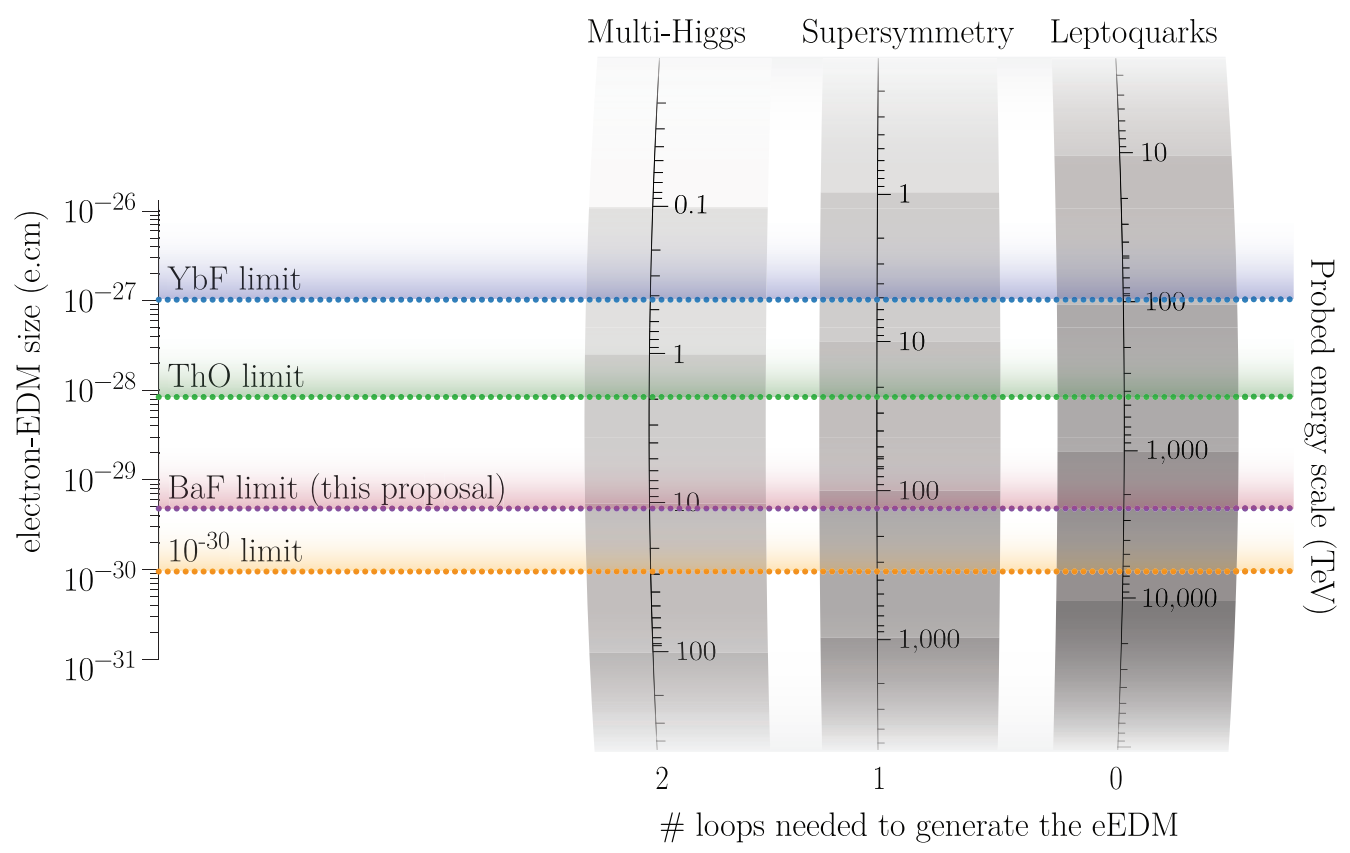

Fig. 1. The experimental upper limits, on the value of eEDM, translated into a limit on the probed energy scale through $d_{e}=e \alpha^{n} \sin \phi\left(m_{e} / \Lambda^{2}\right)$. Here $\alpha$ is the fine-structure constant and $n$ is the number of loops needed to generate the $e$ EDM. $\phi$ is a typical $C P$-violating phase, which is set to the maximum value of $\pi / 2$, and $\Lambda$ is the energy scale that is being probed. The regions above the dotted lines are the energy ranges that are excluded for the indicated classes of models. The models differ in the number of loops required to generate an $e \mathrm{EDM}$. The $e \mathrm{EDM}$ value predicted by the SM, in which the $e \mathrm{EDM}$ only appears at four-loop level, is of the order of $10^{-38} \mathrm{e} \cdot \mathrm{cm}$.

experiments put strong constraints on the validity of the SUSY models and other speculative SM extensions. Improved $e \mathrm{EDM}$ experiments, potentially surpassing the $10^{-30} \mathrm{e} \cdot \mathrm{cm}$ limit, will probe energies approaching the $\mathrm{PeV}$ scale and will thereby contribute to the roadmap of particle physics beyond the LHC.

In this article, we outline our plans to construct and perform an experiment to search for an $e \mathrm{EDM}$ with an intense cold beam of barium monofluoride (BaF) molecules. Our program exploits novel techniques to manipulate and control the quantum states of molecules with electric, magnetic and light fields. Such methods have in recent years promoted diatomic molecules to ultrasensitive probes of particle physics $[10,11]$. We show that this experiment will be sensitive to an $e \mathrm{EDM}$ value of $d_{e}=5 \times 10^{-30} \mathrm{e} \cdot \mathrm{cm}$, which is an improvement by more than one order of magnitude compared to the current upper limit.

\section{The $e$ EDM in atoms and molecules}

Searches for permanent dipole moments of fundamental particles started already before the discovery of $P$ and $C P$ violation, with the investigation of the neutron [12]. Reference [13] contains an early proposal to measure the EDM of the proton. In later years, atomic species such as mercury [14], thallium [15], and xenon [16] were employed in dedicated searches for a permanent EDM and the accompanying $C P$ violation, and the neutron also remained at the focus of attention [17]. For a historical overview of this field of research, we refer to reference [18].

The EDM of composite systems, such as atoms or molecules, can originate in nuclear or electronic contributions (or both), depending on the electronic structure. In addition, in such systems, large enhancement factors can occur for the particle EDMs due to the strong internal electric field near a heavy nucleus. This enhancement has been denoted the effective electric field $\left(E_{\text {eff }}\right)$. In some atoms these enhancements can reach values of $10^{3}-10^{4}$, while in molecules they can go up to $10^{6}$ [19]. Such systems therefore form sensitive testing grounds for probing new sources of $C P$ violation. In paramagnetic systems, $C P$ violation gives rise not only to an $e$ EDM, which is usually the focus of the investigations, but also to semileptonic $C P$-violating interactions between the electrons and the quarks in the atomic nuclei. Theoretical tools are needed to disentangle the different contributions and to relate these to $C P$-violating observables measured at colliders $[20,21]$.

In recent years, experiments with neutral diatomics have set new stringent experimental eEDM limits. The Hinds group at Imperial College, London, uses a supersonic beam of $\mathrm{YbF}$ molecules [8]; $\mathrm{YbF}$ is predicted to have $E_{\text {eff }}$ of $23 \mathrm{GV} / \mathrm{cm}[22]$. The ACME collaboration at Yale/Harvard uses a buffer-gas beam of metastable ThO molecules [9], exploiting the large predicted $E_{\text {eff }}$ in the range of $75-84 \mathrm{GV} / \mathrm{cm}$ [23-28]. The latter experiment was sensitive to frequency shifts of $<6 \mathrm{mHz}$ or an energy shift of $<3 \times 10^{-18} \mathrm{eV}(3 \mathrm{aeV})$, which can be translated, knowing the $E_{\text {eff }}$, to a limit on the $e$ EDM of 
$\left|d_{e}\right|<8.7 \times 10^{-29}$ e.cm. This is currently the most stringent constraint on the $e \mathrm{EDM}$. Other neutral species have also been considered for eEDM searches, such as the $\mathrm{WC}$ [29] and the $\mathrm{PbO}$ [30] molecules. There is also an endeavor to use trapped molecular ions [31] and a study based on the ${ }^{180} \mathrm{Hf}^{19} \mathrm{~F}^{+}$ion has led to a constraint of $\left|d_{e}\right|<1.3 \times 10^{-28}$ e.cm [32].

We aim to use the neutral BaF molecule for an $e \mathrm{EDM}$ measurement. In heavy paramagnetic diatomic molecules, such as $\mathrm{BaF}$ the valence electron is exposed to a huge internal electric field, parametrized by $E_{\text {eff }}$, which enhances the effect of the $e \mathrm{EDM}$ and results in a linear Stark shift. This shift is the experimental signal that we aim to measure [33]. The magnitude of $E_{\text {eff }}$ cannot be measured directly and has to be calculated using electronic structure methods. $\mathrm{BaF}$ has a somewhat smaller predicted $E_{\text {eff }}$ of 6-8.5 GV/cm compared to other molecules used in such experiments, depending on the computational approach [23,34-38]. We are currently performing benchmark quality calculations of the $E_{\text {eff }}$ of $\mathrm{BaF}$, which is needed for the interpretation of the measurements and extraction of the limit on the $e \mathrm{EDM}$. The relativistic coupled cluster approach $(\operatorname{CCSD}(\mathrm{T}))$, considered to be one of the most powerful computational methods, is used in these calculations, and we are also investigating the influence of various computational parameters on the value of obtained $E_{\text {eff }}$. The estimated uncertainty of the final result is expected to be on the order of a few percent [39]. The modest value of $E_{\text {eff }}$ is compensated by the fact that $\mathrm{BaF}$ is lighter than $\mathrm{YbF}$ and ThO, which allows it to be efficiently decelerated in a molecular beam machine of realistic size. Furthermore, unlike ThO, the experiment will be performed in the electronic ground state that has a suitable structure for efficient laser cooling and detection.

\section{Scientific challenges}

\subsection{Boosting the sensitivity of an $e \mathrm{EDM}$ measurement}

The experimental strategy to search for a finite EDM of the electron is illustrated in Figure 2. A superposition of hyperfine substates is created, which builds up a phase difference during the interaction with external electric and magnetic fields. The EDM signal is detectable through a difference in the total accumulated phase for the parallel and the antiparallel orientation of the magnetic and electric fields. The phase difference $\phi_{\text {EDM }}$ due to the EDM is measured by subtracting the average fluorescence count rates for the relative orientations, and is directly proportional to the $e \mathrm{EDM}$ :

$$
\phi_{\mathrm{EDM}}=d_{e}|P| E_{\mathrm{eff}} \tau / \hbar,
$$

where $P$ is the polarisation factor of the molecule and $\tau$ is the coherent interaction time in the interaction zone. The experimental resolution that can be reached depends on the fringe contrast, i.e. on the statistics, on the stability and homogeneity of the applied electric and magnetic

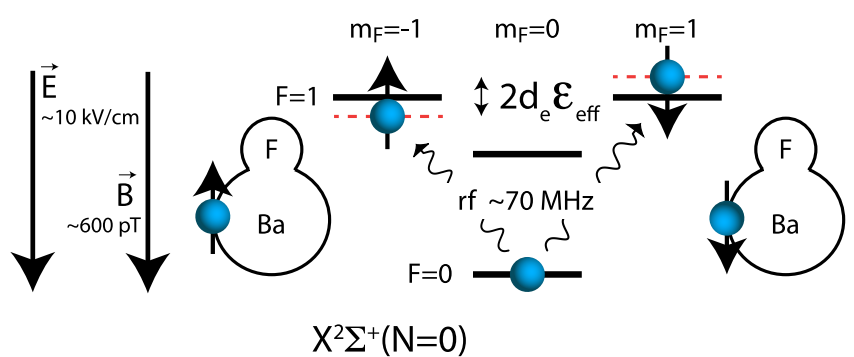

Fig. 2. The ingredients of an $e \mathrm{EDM}$ measurement in $\mathrm{BaF}$ molecules. A superposition of hyperfine substates is created, which builds up a phase difference during the interaction with the electric and magnetic fields. This phase difference, proportional to the product of the $e$ EDM magnitude and the effective electric field inside the molecule, is read out when the superposition is projected back onto the $N=0, F=0$ state.

fields, as well as on the velocity and flux stability of the molecular beam.

The statistical uncertainty $\sigma_{\mathrm{d}}$ of a molecular $e \mathrm{EDM}$ experiment is determined by the interplay of four crucial parameters: the rate of detected molecules $\dot{N}=d N / d t$, the coherent interaction time of the molecules with the electric field $\tau$, the measurement time $T$, and $|P| E_{\text {eff }}$, and is given by

$$
\sigma_{\mathrm{d}}=\frac{\hbar}{e} \frac{1}{2|P| E_{\mathrm{eff}} \tau \sqrt{\dot{N} T}}
$$

It is attractive to use a long coherent interaction time $\tau$, as the sensitivity improves linearly with this parameter. Until recently, increasing the interaction time was accompanied by a significant decrease in the counting rate $\dot{N}$. However, recent advances in decelerating molecular beams [40-43] combined with spectacular progress in molecular laser cooling [44] and the demonstration of intense cryogenic molecular beam sources [45] have opened a route to circumvent this limitation and make long interaction times possible. A similar approach has recently been suggested for the YbF molecule [46].

Our experimental strategy is summarized in Figure 3. The use of advanced slowing and cooling techniques creates a slow and well-collimated molecular beam, which leads to a coherent interaction time one order of magnitude longer than that of competing experiments, without sacrificing the counting rate. Slow $\mathrm{BaF}$ molecules can be detected efficiently by exploiting the closed cycling transition, which further boosts the sensitivity.

We made a conservative estimate of the statistical sensitivity that we can obtain, resulting in $5 \times 10^{-30} \mathrm{e} \cdot \mathrm{cm}$. This is based on the detection of $7 \times 10^{5}$ molecules/shot, with the experiment running at $10 \mathrm{~Hz}$ for a period of $24 \mathrm{~h}$. All essential numbers leading to this estimate are summarized in Table 1. In Section 4 the four main components of the proposed experiment are described in detail, and more information is given to support the numbers in the table. 


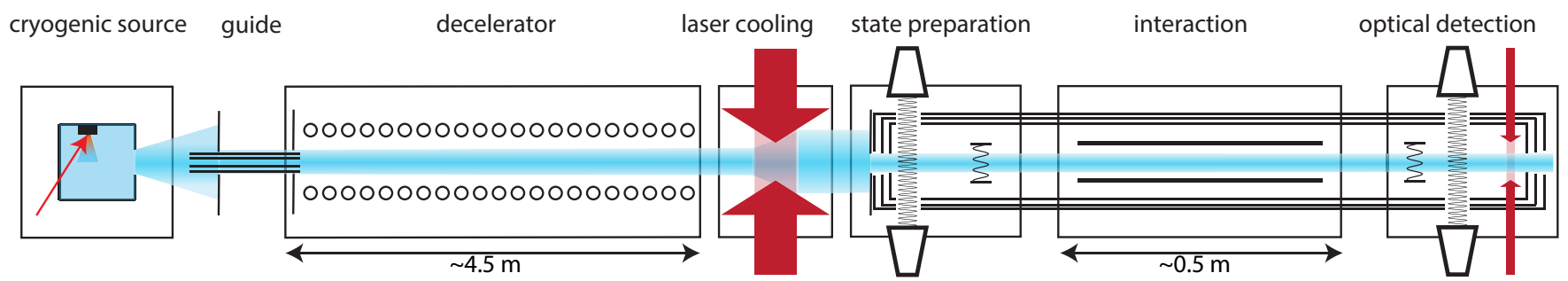

Fig. 3. Schematic overview of the proposed experimental approach. The molecular beam of BaF molecules travels from left to right. A beam with a velocity of $\sim 180 \mathrm{~m} \mathrm{~s}^{-1}$ is created in the cryogenic source, and subsequently decelerated to $\sim 30 \mathrm{~m} \mathrm{~s}^{-1}$ in the Stark decelerator. A laser cooling section reduces the transverse velocity spread of the beam, preventing it from spreading out during the $\sim 15 \mathrm{~ms}$ it takes the molecules to travel through the 0.5 -m-long interaction zone. In the magnetically shielded interaction zone, the $e \mathrm{EDM}$ is probed using Ramsey interferometry in an electric field. Readout of the signal is done by fluorescence detection.

Table 1. The estimate of the number of molecules that can be detected per repetition of the experiment. We aim to run the experiment at $10 \mathrm{~Hz}$.

\begin{tabular}{|c|c|c|c|}
\hline Item & Number & Units & Resulting \# mol./shot \\
\hline \multirow[t]{3}{*}{ Source } & $10^{13}$ & Molecules/shot & \\
\hline & 0.005 & Extraction efficiency from buffer gas cell & \\
\hline & 0.24 & Fraction in $v=0, N=2$ & $5 \times 10^{10}$ from source; $4 \times 10^{9}$ in desired state, \\
\hline \multirow[t]{3}{*}{ Decelerator } & 0.002 & Fraction in velocity acceptance & \\
\hline & 0.3 & Fraction in spatial acceptance & \\
\hline & 0.7 & Efficiency of deceleration relative to guiding & $2 \times 10^{6}, v_{\text {long }}=(30 \pm 6) \mathrm{m} \mathrm{s}^{-1}, v_{\text {trans }}= \pm 5 \mathrm{~m} \mathrm{~s}^{-1}$ \\
\hline \multirow[t]{2}{*}{ Interaction zone } & 0.8 & Transmission and state transfer efficiency & \\
\hline & 1.0 & Detection efficiency & $7 \times 10^{5}$ \\
\hline
\end{tabular}

\subsection{Systematic effects}

The achievable limit on the EDM of the electron depends not only on the statistical uncertainty (see Eq. 2) but also on the control and understanding of experimental procedures, i.e. on systematic uncertainties. The measurement principle and the interaction zone are similar to that of the ongoing molecular EDM experiments such as ACME at Harvard [47] and the YbF experiment at Imperial College [48]. The systematic effects are thus comparable; however, they need to be evaluated explicitly in the context of the current $\mathrm{BaF}$ experiment. We expect to control the combined systematic effects at a level corresponding to an $e$ EDM sensitivity of $10^{-30} \mathrm{e} \cdot \mathrm{cm}$.

In order to suppress systematics all experimental parameters, such as magnetic and electric fields as well as rf and light polarizations, will be switched (reversed) [49]. Such reversals can in practice be achieved with finite accuracy. For example, reversing the electric field may result in a slightly different field strength, which can cause an asymmetry in the experimental conditions. As imperfections in these fields can mimic EDM signals, their mapping and in situ monitoring will be implemented by electro-optical field sensors. Systematic effects that cannot be canceled by parameter reversal need to be carefully addressed individually. These effects will be studied making use of the well controlled molecular beam and, where possible, taken into account by their deliberate exaggeration.
A major distinction of the current approach compared to the previous $e \mathrm{EDM}$ experiments is the long interaction time, which results in increased demands on the magnetic field control. The low value of the magnetic holding field $(\sim 600 \mathrm{pT})$ requires careful shielding of both static and dynamic stray magnetic fields. For this the background magnetic field will be compensated (to about $5 \%$ ) by three sets of large electric current-driven field coils around the interaction zone setup. The interaction zone itself will be embedded in a cylindrical multilayer $\mu$-metal shield for primarily static field compensation. A coaxial, few $\mathrm{mm}$ thick Al shield will be used for AC environmental field suppression, with particular emphasis on the AC field frequencies with periods comparable to the transit time of the molecules.

\section{Experimental set-up}

\subsection{Cryogenic source}

Until now all Stark deceleration experiments (including the experiments with $\mathrm{SrF}$ at the Van Swinderen Institute (VSI) at the University of Groningen [43,50]) have used pulsed supersonic beams as the source. However, over the last decade, several groups have demonstrated a promising new type of molecular-beam source using the cryogenic buffer gas method [45,51-54]. With this method, cold beams of refractory species are generated by ablating a 


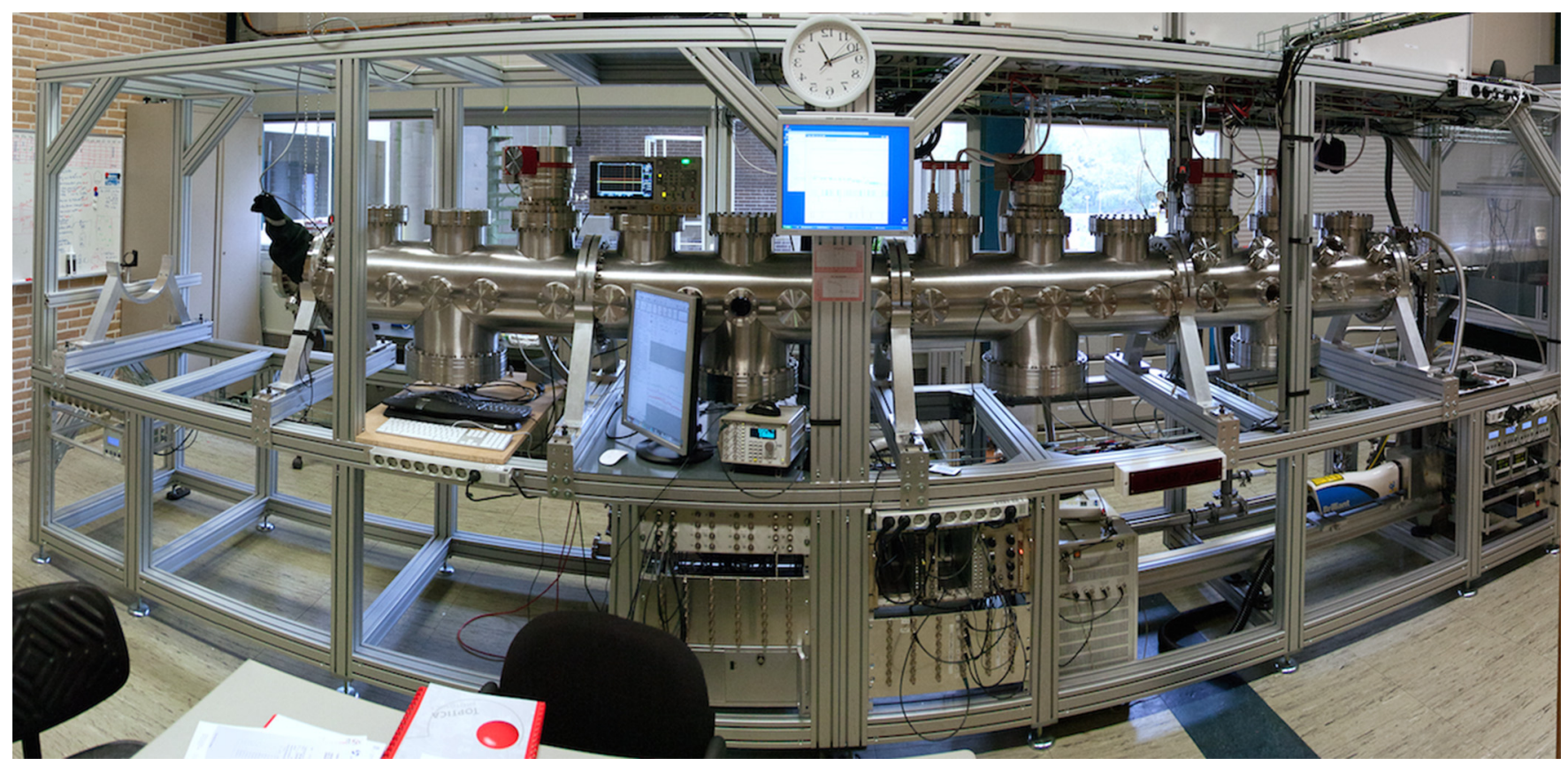

Fig. 4. Panoramic view of the Stark decelerator, operational at VSI in Groningen. This device, with a length of 4.5 meter, was built with the purpose of studying fundamental symmetry violations (note the clock) with slows beams of heavy diatomic molecules.

solid precursor inside a cold cell. This cold cell, with typical dimensions of few centimeters, is filled with helium or neon atoms cooled to $2-20 \mathrm{~K}$ by a closed-cycle cryocooler. The buffer gas in the cell is kept at a specifically tuned atom number density, which is low enough to prevent simple three-body collision cluster formation yet high enough to provide enough collisions for thermalization before the molecules touch the walls of the cold cell. A beam of cold molecules can be formed when the buffer gas and target molecules escape the cell through a fewmillimeter sized opening into a high-vacuum region. When the helium or neon flow through the cell is small, an effusive beam is formed with a relatively low-forward velocity, which scales as the square root of the temperature over the mass of the molecule. At higher flow rates the beam velocity increases, eventually becoming equal to the square root of the temperature over the mass of the atom used as buffer gas, while the extraction efficiency increases as well and the beam becomes more collimated. Beams of slow $\left(<200 \mathrm{~m} \mathrm{~s}^{-1}\right)$ heavy molecules, such as $\mathrm{SrF}, \mathrm{BaF}, \mathrm{YbF}$ and $\mathrm{ThO}$, with a brightness of above $10^{11}$ molecules per steradian per pulse have been reported $[55,56]$.

We will use a cryogenic buffer gas source with a design similar to that demonstrated by Hutzler et al. [56] and Bulleid et al. [52], to create pulses of BaF molecules with a forward velocity of around $180 \mathrm{~m} \mathrm{~s}^{-1}$. At these velocities, the cryogenic source will generate an intense beam of forward-directed molecules that can be effectively captured by our traveling wave decelerator. As a conservative estimate, $10^{13}$ molecules are created per ablation pulse, of which a fraction of $5 \times 10^{-3}$ is extracted into the molecular beam. We therefore expect to be able to create a beam with $5 \times 10^{10}$ molecules in a pulse of $5-10 \mathrm{~ms}$ with a rotational temperature of $2 \mathrm{~K}$, a longitudinal velocity spread of $100 \mathrm{~m} \mathrm{~s}^{-1}$, and a transverse velocity spread of $60 \mathrm{~m} \mathrm{~s}^{-1}$. This intensity is similar to that demonstrated for BaF by Zhou et al. [57], and about the factor 10 smaller than demonstrated for ThO [51].

In order to prevent the buffer gas from entering the decelerator beamline, we will add a series of quadrupole lenses of design similar to those used in the molecular fountain and molecular synchrotron experiments at the Vrije Universiteit, Amsterdam [58,59], which will guide the $\mathrm{BaF}$ molecules from the exit of the cryogenic source to the entrance of the decelerator over a distance of $0.5-1 \mathrm{~m}$. By adding some length, the coupling to the longitudinal phase-space matching of the decelerator can be improved [60]. The guide has a much larger transverse acceptance than the decelerator, so molecules that are lost transversally in the guide would not have been decelerated anyway. The last section of the guide will be used to match the transverse phase-space distribution of the beam to the acceptance of the decelerator [61].

\subsection{Stark deceleration}

To reduce the forward velocity of the molecular beam, we will use a traveling-wave decelerator. This decelerator (depicted in Fig. 4) for heavy diatomic molecules was recently built and taken into operation at VSI. In recent years we have performed a number of experiments to prepare samples of molecules for precision measurements using traveling-wave decelerators [42,43,50,62-64].

The traveling-wave decelerator consists of many ringshaped electrodes that effectively form a 4-mm diameter tube through which the molecular beam travels. Recent results, shown in Figure 5a, illustrate the efficient deceleration of $\mathrm{SrF}$ molecules from a supersonic source with 

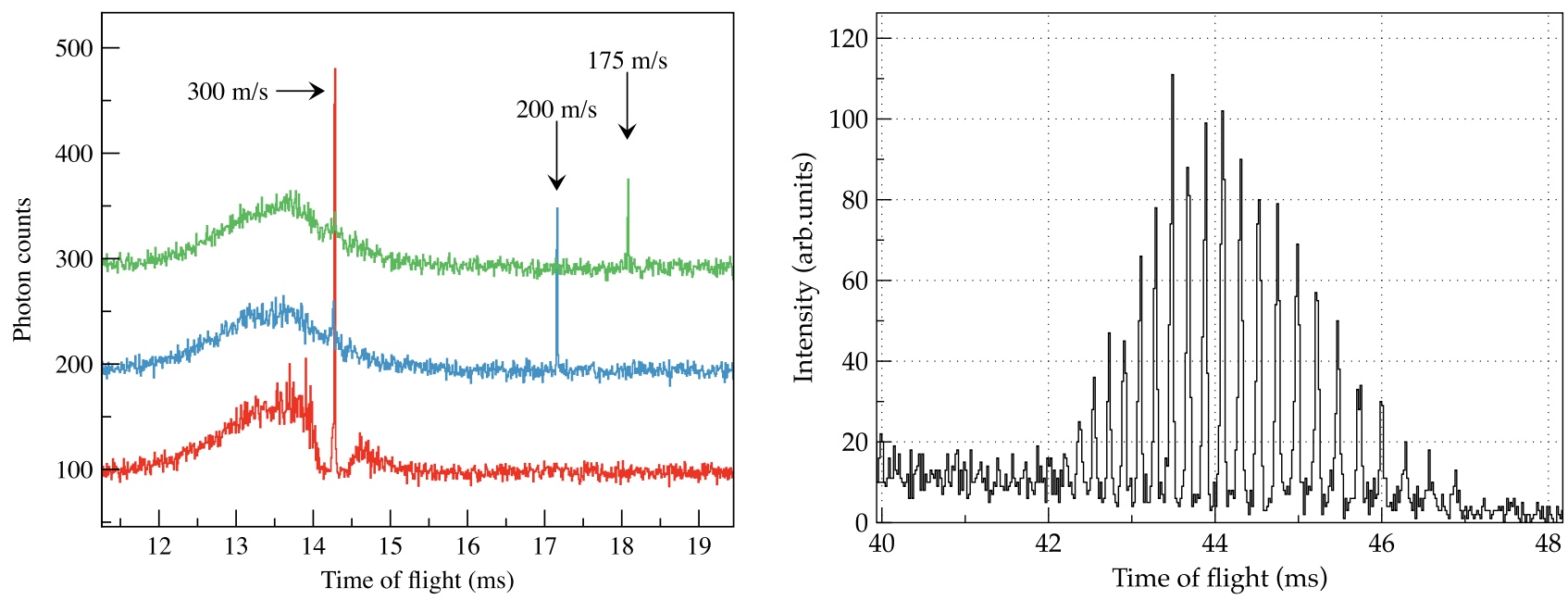

Fig. 5. (a) Time of flight profile showing the arrival time of $\mathrm{SrF}$ molecules captured from a supersonic expansion when a waveform is applied to guide (red) or decelerate (blue and green) the molecules [43,50]. These measurements are performed with a 4-m-long decelerator. The central peak in these plots corresponds to a packet of molecules kept together by the electric fields throughout the deceleration process. (b) A numerical simulation of the time of flight profile illustrates the deceleration of BaF molecules from a cryogenic source, from 180 to $30 \mathrm{~m} \mathrm{~s}^{-1}$, in a $4.5-\mathrm{m}$-long decelerator. Molecules from the long pulse are captured into neighboring traps of the traveling-wave decelerator, resulting in multiple peaks in the time of flight profile. All decelerated molecules can be used for the EDM measurement because they will all propagate, after deceleration, through the interaction zone at $(30 \pm 6) \mathrm{m} \mathrm{s}^{-1}$.

this device. From these results, combined with numerical trajectory simulations, we can deduce the deceleration efficiency for BaF molecules, illustrated in Figure 5b. Due to its length, the VSI decelerator can operate at modest deceleration strengths, which results in the deceleration of the heavy BaF molecules with relatively high efficiency. In this process the molecules are kept together in closely spaced traps of 3 by $6 \mathrm{~mm}$ at all times. As the cryogenic beam emits a rather long pulse, multiple co-moving traps inside the decelerator will be filled. The molecules in these traps will all be decelerated to the same final speed and will all contribute to the $e \mathrm{EDM}$ measurement.

The number of molecules accepted by the decelerator is determined by the required deceleration strength, the applied voltage, and the Stark shift of the molecular state of interest. The Stark shift of the lowest three rotational levels in the electronic ground state of $\mathrm{BaF}$ is shown in Figure 6. The low-field seeking states of $\mathrm{BaF}$ have a turning point in their Stark shift that limits the accepted transverse and longitudinal velocities, which makes deceleration of molecules in the first excited rotational state $(N=1)$ optimal at $5 \mathrm{kV}$, and deceleration of molecules in the $N=2$ state optimal at $10 \mathrm{kV}$. At $5 \mathrm{kV}$, BaF molecules in the $N=1$ state are accepted if they enter the decelerator with a longitudinal velocity within $\pm 5 \mathrm{~m} \mathrm{~s}^{-1}$ of the set velocity and a transverse velocity up to $\pm 3 \mathrm{~m} \mathrm{~s}^{-1}$. At an increased voltage amplitude of $10 \mathrm{kV}, \mathrm{BaF}$ molecules in the $N=2$ state are accepted if they enter the decelerator with a longitudinal velocity within $\pm 8.5 \mathrm{~m} \mathrm{~s}^{-1}$ of the set velocity and a transverse velocity up to $\pm 5 \mathrm{~m} \mathrm{~s}^{-1}$. In order to decelerate a beam of $\mathrm{BaF}$ in the $N=2$ state from 180 to $30 \mathrm{~m} \mathrm{~s}^{-1}$ in $4.5-\mathrm{m}$, the traveling well will be decelerated at $3.5 \mathrm{~km} \mathrm{~s}^{-2}$, which reduces the longitudinal acceptance

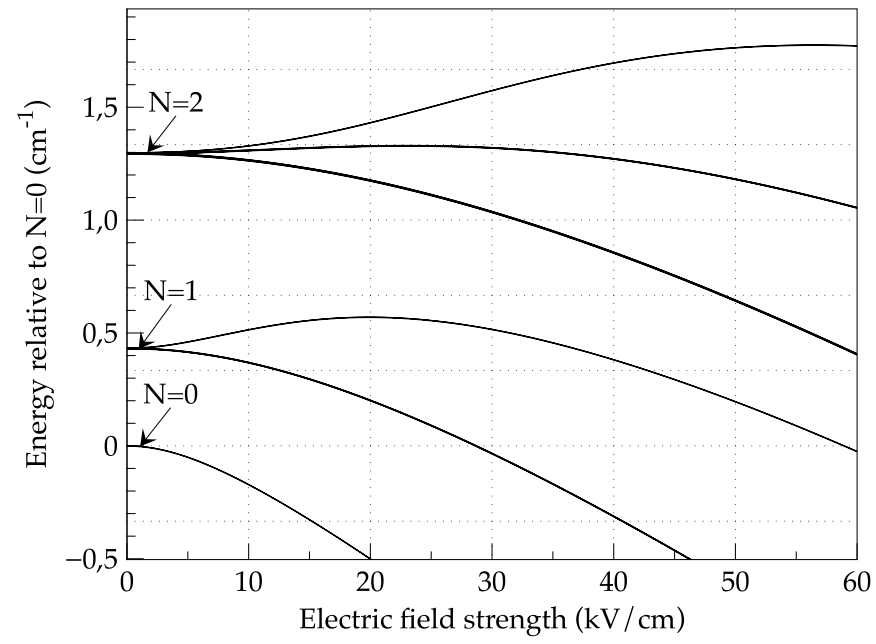

Fig. 6. The lowest three rotational levels within the electronic ground state of the $\mathrm{BaF}$ molecule, as a function of electric field strength. The $N=0$ level is used for the $e \mathrm{EDM}$ measurement; deceleration is most efficient in the $N=2$ level, while lasercooling and detection is best performed in the $N=1$ level.

of the decelerator to $70 \%$ compared to the situation where the well is traveling at a constant speed. Assuming a cryogenic beam of $\mathrm{BaF}$ with a density and velocity spread as discussed in the previous section, we find that a fraction of $4 \times 10^{-4}$ of the cryogenic beam can be decelerated. Consequently, the decelerated beam exiting the traveling-wave decelerator will contain $2 \times 10^{6}$ molecules per pulse, with a longitudinal velocity spread of $\pm 6 \mathrm{~m} \mathrm{~s}^{-1}$ and a transverse velocity spread of $\pm 5 \mathrm{~m} \mathrm{~s}^{-1}$. 


\subsection{Laser cooling}

The slow molecular beam exiting the decelerator diverges due to the transverse velocity spread. To allow the molecular beam to pass through the 50-cm-long EDM measurement zone, laser cooling will be employed to reduce the transverse velocity component. Since laser cooling requires the molecules to be in the $N=1$ level, the molecules will be transferred from the low-field seeking $F=2$ and $F=3$ states of the $N=2$ level to the $F=1$ and $F=2$ states of the $N=1$ using microwave radiation at $25.2 \mathrm{GHz}$, at the beginning of the laser cooling section.

The powerful technique of laser cooling has only recently been extended from atoms to molecules [44,65-68], where the leaky optical cooling cycle leads to challenges comparable to the laser cooling of atoms like $\mathrm{Ba}$ [69]. Some molecules, like $\mathrm{BaF}$, have however a number of properties that make them well-suited for laser cooling, that is (i) a low probability for vibrational excitations upon electronic excitation, (ii) a short excitedstate lifetime [70,71], which is important for a fast cooling cycle, and (iii) convenient transition wavelengths [72-75], where diode lasers with sufficient intensity are available. Recently, a number of investigations have highlighted the possibility of lasercooling of $\mathrm{BaF}$ molecules [76,77]. We have carried out relativistic coupled cluster calculations of the branching ratios and relativistic multireference configuration interaction (MRCI) calculations of the transition dipole moments of the relevant transitions [78]. The obtained Franck-Condon factors are indicated along with the experimentally known energy level structure of $\mathrm{BaF}$ in Figure 7. One main cooling transition and repumping from the vibrational states $v^{\prime \prime}=1$ and $v^{\prime \prime}=2$ is sufficient for the transverse cooling of the BaF beam. The branching to the Delta state $A^{\prime 2} \Delta$ is strongly suppressed due to the small energy separation $[79,80]$. Frequency sidebands will be created on both the main cooling transition and the repump transition to match the hyperfine structure in the ground state [81].

The efficient transverse laser cooling is possible because of the advantageous properties of the slowed $\mathrm{BaF}$ beam at the exit of the decelerator. A single photon at $860 \mathrm{~nm}$, results in a recoil of $\mathrm{BaF}$ of about $3 \mathrm{~mm} \mathrm{~s}^{-1}$. In $2 \mathrm{D}$ optical molasses, the scattering of 2000 photons suffices to dissipate the $5 \mathrm{~m} \mathrm{~s}^{-1}$ transverse velocity at the exit of the decelerator, taking less than $2 \mathrm{~ms}$ at saturation intensity. During the transverse cooling, the molecules travel $\sim 6 \mathrm{~cm}$ and the beam diameter increases to $\sim 1 \mathrm{~cm}$. Saturation intensity in the area of the transverse cooling requires the order of $100 \mathrm{~mW}$ of laser power. Conveniently, the required laser wavelengths and intensities are all available as diode lasers with tapered amplifiers. In order to cover the hyperfine structure splitting of about $25 \mathrm{MHz}$, sidebands to the laser frequency will be generated by electro-optical and acousto-optical modulation or by frequency offset locking of individual diode lasers. The absolute wavelength of the light will be stabilized against the optical frequency comb at the VSI for stable long-term operation. From simulations using the calculated branching ratios, we find that less than $20 \%$ of the molecules are lost to states other than the cooling state.

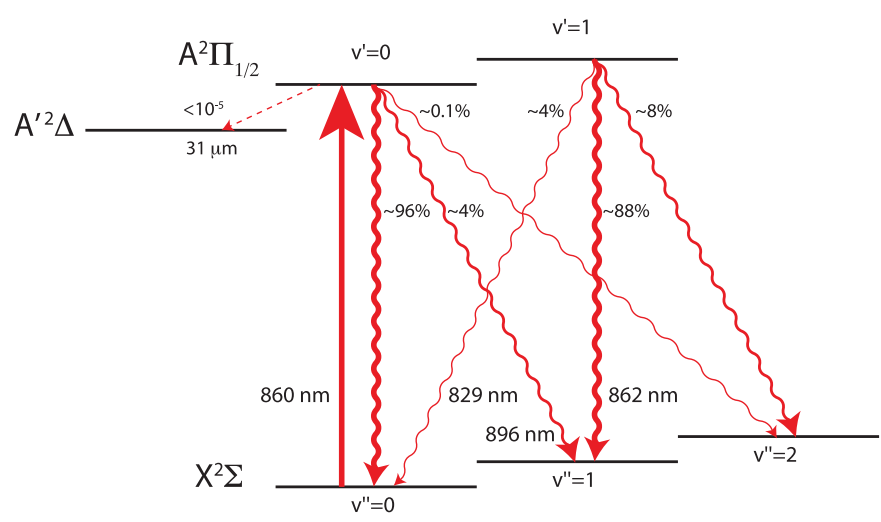

Fig. 7. The lowest vibrational levels within the electronic ground state and electronically excited $A^{2} \Pi_{1 / 2}$ state of the $\mathrm{BaF}$ molecule. The wavelengths of the main transitions are taken from experiment [75]; the branching ratios from the excited state are from calculations performed by this collaboration [78]. Given the low number of photons required for the cooling, two additional lasers repumping molecules from the $v^{\prime \prime}=1$ and $v^{\prime \prime}=2$ are sufficient.

After being laser-cooled, the molecules will be optically pumped into a single hyperfine state of the $N=1$ level, and subsequently transferred to the $F=0$ hyperfine level of the $N=0$ rotational ground state by resonant microwave radiation at $12.6 \mathrm{GHz}$. The combined efficiency of the microwave transfers and optical pumping to the EDM state is estimated to be better than 0.7. Hence we expect the molecular beam emerging from the laser cooling section to have $9 \times 10^{5} \mathrm{~m} \mathrm{~s}^{-1}$, with the transverse velocity reduced to $\pm 0.2 \mathrm{~m} \mathrm{~s}^{-1}$.

\subsection{Interaction zone and measurement}

The interaction zone with a length of $0.5 \mathrm{~m}$ will be magnetically shielded using multiple cylindrical layers of $\mu$-metal [82] with end-caps inside a set of magnetic coils for environmental static field compensation. The external static and dynamic magnetic fields $B_{\text {ext }}$ will be cancelled to $\left|B_{\text {ext }}\right|<50 \mathrm{pT}$. The magnetic field will be monitored with sensitivity at the several $10 \mathrm{fT}$ level [83] with fluxgate (and if needed SERF) magnetometers at fixed locations outside and inside the shielded volume, based on the results of the magnetic field survey at the experiment site. Of particular importance are dynamic field changes at frequencies originating from laboratory technological equipment (such as, $50 \mathrm{~Hz}$ ), the period of which is comparable to the travel time of the molecular beam through the interaction volume. During the measurements, we will use a set of additional coils external to the apparatus in the interaction zone as a feedback system for dynamic field compensation to provide sufficient control of environment-induced magnetic field changes on fast (sub-second) timescales. In order to avoid phase locking of the data acquisition to the frequency of potential perturbations we will adjust the repetition rate for the measurements to be asynchronous with technical frequencies. These measures also prepare the interaction zone for future experiments with slower beams. 
A homogeneous vertical magnetic holding field of $600 \mathrm{pT}$ corresponds to a spin precession angle of $\pi / 4$ when the molecules pass through the interaction zone inside the passive magnetic shield similar to that employed in the ${ }^{129}$ Xe EDM experiment [84]. A relative static field homogeneity of $<10^{-3}$ can be reached with an additional coil system inside the $\mu$-metal shield for higher magnetic multipole compensation. The magnetic field polarity will be regularly reversed throughout the measurements in order to control and check for systematics.

Two parallel plane electrodes, made from a thin-low conductance material deposited on glass, provide an electric field of order $10 \mathrm{kV} / \mathrm{cm}$ parallel to the magnetic holding field. For control of systematics the electric field strength will be monitored by a recently developed ultralow frequency electro-optic sensor [85]. The molecular state preparation and state detection regions are located near both ends of and inside the electric field volume. This yields quantitative state identification in $\mathrm{BaF}$ via fluorescence detection. For the EDM measurements, we will repeatedly reverse the electric field on timescales that are long compared to the coherence time. This procedure will include settling times for field stabilization.

After the molecules pass the electric field region the molecular superposition state is projected back onto the first excited rotational level, from where $\sim 10^{3}$ photons can be scattered per molecule. These photons are imaged with $1 \%$ efficiency onto a position-sensitive low-noise single-photon detector. A cooled EMCCD camera, with a quantum efficiency of $50 \%$ at $860 \mathrm{~nm}$, is capable of quantitatively detecting, without pileup, the high instantaneous photon rates of above $10^{7}$ photons per shot, which we expect at the detector. Thus, 5 photons on average will be detected for each molecule, which results in a detection efficiency of close to unity.

\section{Future perspectives}

The proposed use of a slow, intense, and cold molecular beam is, with the current level of technology, a promising approach to measure the $e \mathrm{EDM}$ and will allow for a measurement that is more than one order of magnitude below the current limit. The great sensitivity of the proposed scheme (mainly) stems from the interaction time that is over 10 times longer than used in ongoing experiments. We believe that in future experiments the interaction times can be increased even further. The interaction time in a horizontal beam machine is limited to about $15 \mathrm{~ms}$, as with longer times the beam will fall under gravity and will miss the detection zone. An elegant way to circumvent this is by using a vertical beam machine in fountain geometry. We have recently demonstrated the first-ever fountain for molecules, which enables the study of ammonia molecules in free fall for up to $266 \mathrm{~ms}$ [58]. A crucial part of this machine is the travelling-wave decelerator that enables us to manipulate the motion of polar molecules virtually without losses. Using a combination of quadrupole lenses and bunching elements, the slow ammonia beam exiting the decelerator is shaped such that it has a large position spread and a small velocity spread when the molecules are in free fall, while being strongly focused at the detection region. Using a similar lens system to focus the laser cooled $\mathrm{BaF}$ beam, it should be possible to create a (quasi$\mathrm{cw}$ ) fountain offering interaction times of up to a second without significant loss in flux. Ultimately, the most sensitive experiment could be based on optically trapped molecules. However, significant hurdles still have to be overcome to realize this: a means to accumulate a sufficiently large number of molecules has to be demonstrated as well as cooling to significantly lower temperatures than can currently be obtained.

This work is part of the research programme "Physics beyond the Standar Model with cold molecules" with project number 166, which is financed by the Netherlands Organisation for Scientific Research (NWO).

\section{Author contribution statement}

The manuscript was drafted by H.L.B., A.B., S.H., K.J., R.G.E.T., W.U. and L.W. All authors have contributed through discussions and corrections to the final manuscript.

Open Access This is an open access article distributed under the terms of the Creative Commons Attribution License (http://creativecommons.org/licenses/by/4.0), which permits unrestricted use, distribution, and reproduction in any medium, provided the original work is properly cited.

\section{References}

1. G. Aad et al., Phys. Lett. B 716, 1 (2012)

2. S. Chatrchyan et al., Phys. Lett. B 716, 30 (2012)

3. G. Bertone, D. Hooper, J. Silk, Phys. Rep. 405, 279 (2005)

4. R. Aaij et al., Int. J. Mod. Phys. A 30, 1530022 (2015)

5. M. Pospelov, A. Ritz, Ann. Phys. 318, 119 (2005)

6. V. Cirigliano, W. Dekens, J. de Vries, E. Mereghetti, Phys. Rev. D 94, 016002 (2016)

7. A. Czarnecki, W.J. Marciano, Adv. Ser. Direct. High Energy Phys. 20, 11 (2009)

8. J.J. Hudson, D.M. Kara, I.J. Smallman, B.E. Sauer, M.R. Tarbutt, E.A. Hinds, Nature 473, 493 (2011)

9. J. Baron, W.C. Campbell, D. DeMille, J.M. Doyle, G. Gabrielse, Y.V. Gurevich, P.W. Hess, N.R. Hutzler, E. Kirilov, I. Kozyryev, B.R. O'Leary, C.D. Panda, M.F. Parsons, E.S. Petrik, B. Spaun, A.C. Vutha, A.D. West, Science 343, 269 (2014)

10. D. DeMille, Phys. Today 68, 34 (2015)

11. D. DeMille, J.M. Doyle, A.O. Sushkov, Science 357, 990 (2017)

12. E.M. Purcell, N.F. Ramsey, Phys. Rev. 78, 807 (1950)

13. P.G.H. Sandars, Phys. Rev. Lett. 19, 1396 (1967)

14. W.C. Griffith, M.D. Swallows, T.H. Loftus, M.V. Romalis, B.R. Heckel, E.N. Fortson, Phys. Rev. Lett. 102, 101601 (2009)

15. B.C. Regan, E.D. Commins, C.J. Schmidt, D. DeMille, Phys. Rev. Lett. 88, 071805 (2002)

16. M.A. Rosenberry, T.E. Chupp, Phys. Rev. Lett. 86, 22 (2001) 
17. C.A. Baker, D.D. Doyle, P. Geltenbort, K. Green, M.G.D. van der Grinten, P.G. Harris, P. Iaydjiev, S.N. Ivanov, D.J.R. May, J.M. Pendlebury, J.D. Richardson, D. Shiers, K.F. Smith, Phys. Rev. Lett. 97, 131801 (2006)

18. K. Jungmann, Ann. Phys. 525, 550 (2013)

19. V.A. Dzuba, V.V. Flambaum, Int. J. Mod. Phys. E 21, $1230010(2012)$

20. J.S.M. Ginges, V.V. Flambaum, Phys. Rep. 397, 63 (2004)

21. J. Engel, M.J. Ramsey-Musolf, U. van Kolck, Prog. Part. Nuc. Phys. 71, 74 (2013)

22. M. Abe, G. Gopakumar, M. Hada, B.P. Das, H. Tatewaki, D. Mukherjee, Phys. Rev. A 90, 022501 (2014)

23. E.R. Meyer, J.L. Bohn, Phys. Rev. A 78, 010502 (2008)

24. L.V. Skripnikov, A.N. Petrov, A.V. Titov, J. Chem. Phys. 139, 221103 (2013)

25. T. Fleig, M.K. Nayak, J. Mol. Spectrosc. 300, 16 (2014)

26. L.V. Skripnikov, A.V. Titov, J. Chem. Phys. 142, 024301 (2015)

27. L.V. Skripnikov, J. Chem. Phys. 145, 214301 (2016)

28. M. Denis, T. Fleig, J. Chem. Phys. 145, 214307 (2016)

29. J. Lee, J. Chen, L.V. Skripnikov, A.N. Petrov, A.V. Titov, N.S. Mosyagin, A.E. Leanhardt, Phys. Rev. A 87, 022516 (2013)

30. S. Eckel, P. Hamilton, E. Kirilov, H.W. Smith, D. DeMille, Phys. Rev. A 87, 052130 (2013)

31. H. Loh, K.C. Cossel, M.C. Grau, K.-K. Ni, E.R. Meyer, J.L. Bohn, J. Ye, E.A. Cornell, Science 342, 1220 (2013)

32. W.B. Cairncross, D.N. Gresh, M. Grau, K.C. Cossel, T.S. Roussy, Y. Ni, Y. Zhou, J. Ye, E.A. Cornell, Phys. Rev. Lett. 119, 153001 (2017)

33. E.A. Hinds, Phys. Scr. 1997, 34 (1997)

34. M.G. Kozlov, L.N. Labzowsky, J. Phys. B 28, 1993, (1995)

35. M.K. Nayak, R.K. Chaudhuri, J. Phys. B 39, 1231 (2006)

36. M. Fukuda, K. Soga, M. Senami, A. Tachibana, Phys. Rev. A 93, 012518 (2016)

37. K. Gaul, R. Berger, J. Chem. Phys. 147, 014109 (2017)

38. M. Abe, V.S. Prasannaa, B.P. Das, Phys. Rev. A 97, 032515 (2018)

39. P.A.B. Haase, M. Iliaš, E. Eliav, P. Aggarwal, H.L. Bethlem, A. Borschevsky, M. Denis, K. Esajas, Y. Hao, S. Hoekstra, K. Jungmann, T.B. Meijknecht, M.C. Mooij, R.G.E. Timmermans, W. Ubachs, L. Willmann, A. Zapara, in prep.

40. A. Osterwalder, S.A. Meek, G. Hammer, H. Haak, G. Meijer, Phys. Rev. A 81, 051401 (2010)

41. N.E. Bulleid, R.J. Hendricks, E.A. Hinds, S.A. Meek, G. Meijer, A. Osterwalder, M.R. Tarbutt, Phys. Rev. A 86, 021404 (2012)

42. M. Quintero-Pérez, T.E. Wall, S. Hoekstra, H.L. Bethlem, J. Mol. Spectrosc. 300, 112 (2014)

43. J.E. van den Berg, S.C. Mathavan, C. Meinema, J. Nauta, T.H. Nijbroek, K. Jungmann, H.L. Bethlem, S. Hoekstra, J. Mol. Spectrosc. 300, 22 (2014)

44. E.S. Shuman, J.F. Barry, D. DeMille, Nature 467, 820 (2010)

45. D. Patterson, J.M. Doyle, J. Chem. Phys. 126, 154307 (2007)

46. J. Lim, J.R. Almond, M.A. Trigatzis, J.A. Devlin, N.J. Fitch, B.E. Sauer, M.R. Tarbutt, E.A. Hinds, Phys. Rev. Lett. 120, 123201 (2018)

47. J. Baron, W.C. Campbell, D. DeMille, J.M. Doyle, G. Gabrielse, Y.V. Gurevich, P.W. Hess, N.R. Hutzler, E. Kirilov, I. Kozyryev, B.R. O'Leary, C.D. Panda, M.F.
Parsons, B. Spaun, A.C. Vutha, A.D. West, E.P. West, New J. Phys. 19, 073029 (2017)

48. D.M. Kara, I.J. Smallman, J.J. Hudson, B.E. Sauer, M.R. Tarbutt, E.A. Hinds, New J. Phys. 14, 103051 (2012)

49. J.J. Hudson, M.R. Tarbutt, B.E. Sauer, E.A. Hinds, New J. Phys. 16, 013005 (2014)

50. S.C. Mathavan, A. Zapara, Q. Esajas, S. Hoekstra, ChemPhysChem 17, 3709 (2016)

51. N.R. Hutzler, M.F. Parsons, Y.V. Gurevich, P.W. Hess, E. Petrik, B. Spaun, A.C. Vutha, D. DeMille, G. Gabrielse, J.M. Doyle, Phys. Chem. Chem. Phys. 13, 18976 (2011)

52. N.E. Bulleid, S.M. Skoff, R.J. Hendricks, B.E. Sauer, E.A. Hinds, M.R. Tarbutt, Phys. Chem. Chem. Phys. 15, 12299 (2013)

53. S.E. Maxwell, N. Brahms, R. DeCarvalho, D.R. Glenn, J.S. Helton, S.V. Nguyen, D. Patterson, J. Petricka, D. DeMille, J.M. Doyle, Phys. Rev. Lett. 95, 173201 (2005)

54. L.D. van Buuren, C. Sommer, M. Motsch, S. Pohle, M. Schenk, J. Bayerl, P.W.H. Pinkse, G. Rempe, Phys. Rev. Lett. 102, 033001 (2009)

55. S.M. Skoff, R.J. Hendricks, C.D.J. Sinclair, J.J. Hudson, D.M. Segal, B.E. Sauer, E.A. Hinds, M.R. Tarbutt, Phys. Rev. A 83, 023418 (2011)

56. N.R. Hutzler, H.-I. Lu, J.M. Doyle, Chem. Rev. 112, 4803 (2012)

57. Y. Zhou, D.D. Grimes, T.J. Barnum, D. Patterson, S.L. Coy, E. Klein, J.S. Muenter, R.W. Field, Chem. Phys. Lett. 640, 124 (2015)

58. C. Cheng, A.P.P. van der Poel, P. Jansen, M. QuinteroPérez, T.E. Wall, W. Ubachs, H.L. Bethlem, Phys. Rev. Lett. 117, 253201 (2016)

59. P.C. Zieger, S.Y.T. van de Meerakker, C.E. Heiner, H.L. Bethlem, A.J.A. van Roij, G. Meijer, Phys. Rev. Lett. 105, 173001 (2010)

60. M.I. Fabrikant, T. Li, N.J. Fitch, N. Farrow, J.D. Weinstein, H.J. Lewandowski, Phys. Rev. A 90, 033418 (2014)

61. S.Y.T. van de Meerakker, H.L. Bethlem, N. Vanhaecke, G. Meijer, Chem. Rev. 112, 4828 (2012)

62. J.E. van den Berg, S.H. Turkesteen, E.B. Prinsen, S. Hoekstra, Eur. Phys. J. D 66, 235 (2012)

63. M. Quintero-Pérez, P. Jansen, T.E. Wall, J.E. van den Berg, S. Hoekstra, H.L. Bethlem, Phys. Rev. Lett. 110, 133003 (2013)

64. P. Jansen, M. Quintero-Pérez, T.E. Wall, J.E. van den Berg, S. Hoekstra, H.L. Bethlem, Phys. Rev. A 88, 043424 (2013)

65. M.T. Hummon, M. Yeo, B.K. Stuhl, A.L. Collopy, Y. Xia, J. Ye, Phys. Rev. Lett. 110, 143001 (2013)

66. V. Zhelyazkova, A. Cournol, T.E. Wall, A. Matsushima, J.J. Hudson, E.A. Hinds, M.R. Tarbutt, B.E. Sauer, Phys. Rev. A 89, 053416 (2014)

67. J.F. Barry, D.J. McCarron, E.B. Norrgard, M.H. Steinecker, D. DeMille, Nature 512, 286 (2014)

68. I. Kozyryev, L. Baum, K. Matsuda, B.L. Augenbraun, L. Anderegg, A.P. Sedlack, J.M Doyle, Phys. Rev. Lett. 118, 173201 (2017)

69. S. De, U. Dammalapati, K. Jungmann, L. Willmann, Phys. Rev. A 79, 041402 (2009)

70. L.-E. Berg, T. Olsson, J.-C. Chanteloup, A. Hishikawa, P. Royen, Mol. Phys. 79, 721 (1993)

71. L.-E. Berg, N. Gador, D. Husain, H. Ludwigs, P. Royen, Chem. Phys. Lett. 287, 89 (1998) 
72. C. Effantin, J. D'Incan, A. Bernard, G. Fabre, R. Stringat, J. Vergès, R. Barrow, J. Phys. Colloq. 48, 673 (1987)

73. C. Effantin, A. Bernard, J. d'Incan, G. Wannous, J. Vergès, R.F. Barrow, Mol. Phys. 70, 735 (1990)

74. A. Bernard, C. Effantin, J. d'Incan, J. Vergès, R.F. Barrow, Mol. Phys. 70, 747 (1990)

75. A. Bernard, C. Effantin, E. Andrianavalona, J. Vergès, R.F. Barrow, J. Mol. Spectrosc. 152, 174 (1992)

76. T. Chen, W. Bu, B. Yan, Phys. Rev. A 94, 063415 (2016)

77. T. Chen, W. Bu, B. Yan, Phys. Rev. A 96, 053401 (2017)

78. Y. Hao, L.F. Pašteka, L.Visscher; and the NL-eEDM Collaboration: P. Aggarwal, H.L. Bethlem, A. Borschevsky, M. Denis, K. Esajas, P.A.B. Haase, S. Hoekstra, K. Jungmann, T. B. Meijknecht, M. C. Mooij, R. G. E. Timmermans, W. Ubachs, L. Willmann, A. Zapara, in prep.
79. R.F. Barrow, A. Bernard, C. Effantin, J. D'Incan, G. Fabre, A. El Hachimi, R. Stringat, J. Vergès, Chem. Phys. Lett. 147, 535 (1988)

80. S. Kang, F. Kuang, G. Jiang, J. Du, Mol. Phys. 114, 810 (2016)

81. W.E. Ernst, J. Kändler, T. Törring, J. Chem. Phys. 84, 4769 (1986)

82. E. Paperno, H. Koide, I. Sasada, J. Appl. Phys. 87, 5959 (2000)

83. D. Budker, M. Romalis, Nat. Phys. 3, 227 (2007)

84. F. Allmendinger, P. Blümler, M. Doll, O. Grasdijk, W. Heil, K. Jungmann, S. Karpuk, H.-J. Krause, A. Offenhäusser, M. Repetto, U. Schmidt, Y. Sobolev, K. Tullney, L. Willmann, S. Zimmer, Eur. Phys. J. D 71, $98(2017)$

85. O. Grasdijk, Search for the permanent electric dipole moment of 129Xe (University of Groningen, The Netherlands, 2018) 